\title{
MULTI-LEVEL SEQUENCE MINING BASED ON GSP
}

\author{
Michal ŠEBEK, Martin HLOSTA, Jan KUPČÍK, Jaroslav ZENDULKA, Tomáš HRUŠKA \\ Faculty of Information Technology, IT4Innovations Centre of Excellence, Brno University of Technology, \\ Božetěchova 1/2, 61266 Brno, Czech Republic, tel.: +420 541141100 , \\ e-mail: isebek@fit.vutbr.cz, ihlosta@ fit.vutbr.cz, ikupcik@ fit.vutbr.cz, zendulka@ fit.vutbr.cz, hruska@ fit.vutbr.cz
}

\begin{abstract}
Mining sequential patterns is an important problem in the field of data mining and many algorithms and optimization techniques have been published to deal with that problem. The GSP algorithm, which is one of them, can be used for mining sequential patterns with some additional constraints. In this paper, we propose a new algorithm for mining multi-level sequential patterns based on GSP. The idea is that if a more general item appears in a pattern, the pattern has higher or at least the same support as the one containing the corresponding specific item. However, too generalized sequence patterns are not important for user. In our algorithm generalization uses a selective method based on information content of patterns. This allows us to mine more patterns with the same minimal support threshold and to reveal new potentially useful patterns.
\end{abstract}

Keywords: multi-level sequence pattern mining, GSP, taxonomy

\section{INTRODUCTION}

A great amount of data is being collected and stored in databases for various purposes. After years the size of these databases became enormous. In such amount of data hidden and interesting patterns may occur. Data mining, also known as knowledge discovery in databases, is a field of study to find these patterns in data. One of the typical data mining tasks is discovery of frequent patterns and association analysis. The latter one is seeking for rules in the form antecedent $\rightarrow$ consequent, which occur in the data often enough and are so called strong. The strength means that the conditional probability $P$ (consequent $\mid$ antecedent) is above a given threshold. Association analysis is abundantly used in market basket analysis to discover habits of customers. Its results can be employed for product recommendation. An example of such a rule can be $T V \rightarrow$ DVD player telling that when customers buy a TV it's likely that a DVD player will appear in their market basket too.

If time information occurs in the data, not only association analysis but also a sequential patterns mining task can be specified. In such a case, the task is to reveal frequently occurring sequences in a sequence database. According to the previously mentioned market basket analysis, an example of such a sequence pattern is $\left\langle T V D V D_{-}\right.$player $\rangle$. This means that customers very often buy a TV set and later a DVD player.

In addition, one or multiple taxonomies of items can be stored in a database. This allows mining patterns with items on different levels of a hierarchy specified by the taxonomies. Then, the resulting pattern set can contain items from more general levels that might not be directly stored in the database. This can provide analysts better insight of the data and reveal patterns that they wouldn't get with a pure sequence mining algorithm. It also allows us to set the minimum support parameter to a higher value and to get results containing more database sequences, because sequences with more general items have at least the same support as their more specific variants. Some algorithms can only find patterns, where all items in one pattern are on the same level of the hierarchy. They are referred to as intra-level patterns. If items of a pattern can be on different levels of a hierarchy, they are called inter-level or levelcrossing patterns. Our method is capable of revealing both intra-level and inter-level sequence patterns.

The remainder of the paper is organized as follows. In Section 2 there is formally defined the problem of mining sequential patterns with taxonomies and terms related to that problem and to information theory. Algorithms related to our work are described in Section 3. The proposed method for mining sequential patterns with taxonomies is described in Section 4. In Section 5 we present performance evaluation of our method.

\section{PROBLEM DEFINITION}

In this section we present definitions of notions for mining sequential patterns with taxonomies from databases and the problem is formally defined. The section begins with terms related to sequential pattern mining, then focuses on taxonomy and ends with terms of the information theory necessary for our algorithm.

\subsection{Sequence pattern mining}

The following definitions are based on established definitions from sequence pattern mining in $[1,3,7]$.

Definition 2.1. (Itemset) Let $I=\left\{i_{1}, i_{2}, i_{3}, \ldots, i_{k}\right\}$ be a set of items. Then an itemset is a nonempty subset of I.

Definition 2.2. (Sequence) A sequence is an ordered list of itemsets. A sequence $s$ is denoted by $\left\langle s_{1} s_{2} s_{3} \ldots s_{n}\right\rangle$, where $s_{j}$ is an itemset. $s_{j}$ is also called an element of the sequence. In the context of sequence patterns mining, the length of a sequence is defined as the number of instances of items in the sequence [1]. A sequence of length $l$ is called an 1-sequence. The sequence $\alpha=\left\langle a_{1} a_{2} \ldots a_{n}\right\rangle$ is a subsequence of the sequence $\beta=\left\langle b_{1} b_{2} \ldots b_{m}\right\rangle$ if there exist integers $1 \leq j_{1}<j_{2}<\cdots<j_{n} \leq m$ such that $a_{1} \subseteq b_{j_{1}}, a_{2} \subseteq$ $b_{j_{2}}, \ldots, a_{n} \subseteq b_{j_{n}}$. We denote it $\alpha \sqsubseteq \beta$ and $\beta$ is a supersequence of $\alpha[1]$. 
Example 2.1. In examples, for improving the readability, we use well established convention from sequence pattern mining for denoting elements of sequences [1]. An element that has $m$ items is denoted as $\left(i_{1} i_{2} \ldots i_{m}\right)$ where $m \geq 1$. If an element contains only one item, the braces are omitted for brevity.

Based on these definitions, given $I=\{a, b, c, d, e\}$ an example of an itemset is $\{a, b, d\}$, example of a sequence is $\langle a(a b)(c e) d(c d e)\rangle$. This sequence has 5 elements and 9 items thus it's length is 9 and it's called a 9-sequence.

Definition 2.3. (Sequence database) A sequence database $D$ is a set of tuples $\langle S I D, s\rangle$, where SID is the unique identification of a sequence and $s$ is the sequence. The support of the sequence $s_{1}$ is defined as a number of tuples in the database in which sequences are supersequences of $s_{1}$. Formally, the support of sequence $s_{1}$ is

$\operatorname{support}\left(s_{1}\right)=\left|\left\{\langle S I D, s\rangle \mid(\langle S I D, s\rangle \in D) \wedge\left(s_{1} \sqsubseteq s\right)\right\}\right|$.

Definition 2.4. A frequent sequence is defined as sequence $s$ if support $(s) \geq$ min_sup which is the minimum support threshold provided by the user. A frequent sequence is called sequence pattern.

Based on the defined terms, we can formally define the problem of mining sequential patterns as follows: Given a sequence database $D$ and the minimal support threshold min_sup, the problem is to find all the frequent sequences with support greater or equal than min_sup, that is frequent sequences.

\subsection{Taxonomy}

Definition 2.5. (Taxonomy) taxonomy structure is a rooted tree. A rooted tree $T=(V, E)$ is a tree with one vertex $r \in V$ chosen as its root. We refer to a vertex of a rooted tree as a node. For each node $v$ in a tree, let $U P(v)$ be the simple unique path from $v$ to $r$. If $U P(v)$ has exactly $k$ edges then the level of $v$ is $k$ for $k \geq 0$. The level of the root is 0 . The height of a taxonomy is the greatest level in the tree. The parent of $v \neq r$, formally parent $(v)$, is neighbour of $v$ on $U P(v)$, and for each node $v \in V, v \neq r$ there exists a set of its ancestors defined as:

$\operatorname{ancestors}(v)=\{x \mid x \in U P(v), x \neq v\}$.

The parent of $r$ and the ancestors of $r$ are not defined. We say if $v$ is the parent of $u$ then $u$ is a child of $v$. A leaf is a node having no child [13].

In every taxonomy structure there exists $a$ is-a relation which is defined as follows:

is-a $: V \times V: \equiv\{(a, b) \mid b \in$ ancestors $(a)\}$

Let $\tau$ be a nonempty set of taxonomy structures such that nodes in taxonomy structures represent items and each item $i \in I$ appears in exactly one taxonomy structure $t \in \tau$. Notice that we do not require that items need to be only leaf nodes. In addition we refer to any ancestor of node representing item $x$ a generalized item of item $x$.
Example 2.2. An example of a taxonomy structure is depicted in figure 1 that represents some food products. The height of this taxonomy is 2 and all its leaves has the same level. The food represents the root node and is ancestor of all other nodes. The dairy product is parent and ancestor of the cheese and in terms of the relation is-a the cheese is-a dairy product and also it is-a food.

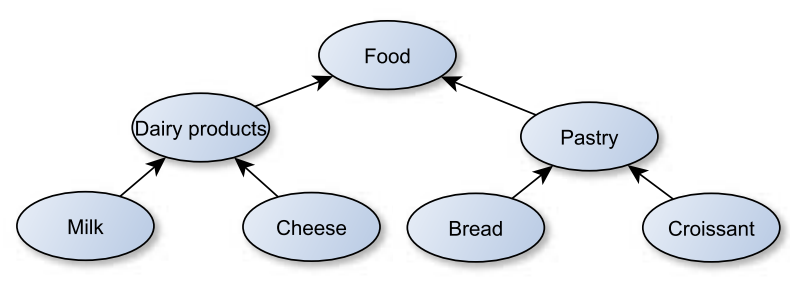

Fig. 1 Example of a food taxonomy structure

Definition 2.6. (Element parents) Given an element $e=$ $\left\{i_{1}, i_{2}, \ldots, i_{n}\right\}$, an element parents of the element $e$ is a set of the elements which are the same as e but exactly one of the items is generalized. This is defined as

$$
\begin{aligned}
\text { parents }_{e l}(e)= & \left\{e \backslash\left\{i_{k}\right\} \cup\left\{\text { parent }\left(i_{k}\right)\right\} \mid i_{k} \in e\right. \\
& \left.\wedge \text { parent }\left(i_{k}\right) \notin e\right\} .
\end{aligned}
$$

Based on the definition of element parents, we can define the sequence parents and ancestors.

Definition 2.7. (Sequence parents and ancestors) Given a sequence $s=\left\langle e_{1} e_{2} \ldots e_{n}\right\rangle$, where $e_{k}$ is element, the sequence parents of $s$ is the set of sequences that are the same as the sequence s but exactly one of their elements is replaced by one of its element parents. Formally,

$$
\begin{aligned}
\text { parents }_{\text {seq }}(s)= & \left\{\left\langle f_{1} f_{2} \ldots f_{n}\right\rangle \mid f_{k} \in \text { parents }_{e l}\left(e_{k}\right)\right. \\
& \wedge 1 \leq k \leq n \\
& \left.\wedge \forall l \neq k, 1 \leq l \leq n: e_{l}=f_{l}\right\} .
\end{aligned}
$$

For a given $\tau$, a root sequence is a sequence consisting of elements with items corresponding to root nodes only. The sequence parents of a root sequence is empty set. Based on the definition of the sequence parents, the sequence ancestors of the sequence $s$, ancestors $s_{s e q}(s)$ is defined as follows:

$$
\begin{aligned}
\operatorname{ancestors}_{\text {seq }}(s)= & M_{i}, \text { if } M_{i+1}=M_{i}, \text { where } \\
M_{0}= & \text { parents }_{\text {seq }}(s) \\
M_{i+1}= & M_{i} \cup\left\{\text { parents }_{\text {seq }}(x) \mid x \in M_{i}\right\} \\
& \text { for } i \geq 0 .
\end{aligned}
$$

Example 2.3. These notions can be well understood by looking at the figure 2 with two taxonomy structures for two root items $A$ and $B$. Item $A$ is an ancestor and a parent of items $a$ and $a^{\prime}$. The bottom of the figure depicts sequence ancestors for the sequence $\left\langle a\left(a^{\prime} b\right)\right\rangle$. The arrows in the figure represent which nodes are parents to the selected node. 
Sequences $\left\langle a\left(a^{\prime} B\right)\right\rangle,\left\langle A\left(a^{\prime} b\right)\right\rangle,\langle a(A b)\rangle$ are parents of the sequence $\left\langle a\left(a^{\prime} b\right)\right\rangle$, because exactly one of their elements is the element parent of an element in $\left\langle a\left(a^{\prime} b\right)\right\rangle$, for example $\left(a^{\prime} B\right)$ is the element parent of the $\left(a^{\prime} b\right)$. All of the sequences in nodes above sequence $\left\langle a\left(a^{\prime} b\right)\right\rangle$ are its sequence ancestors and the sequence $\langle A(A B)\rangle$ is its root sequence.
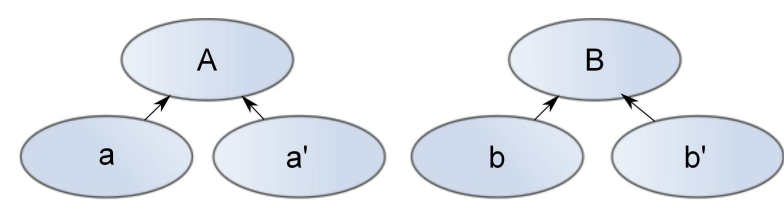

a)

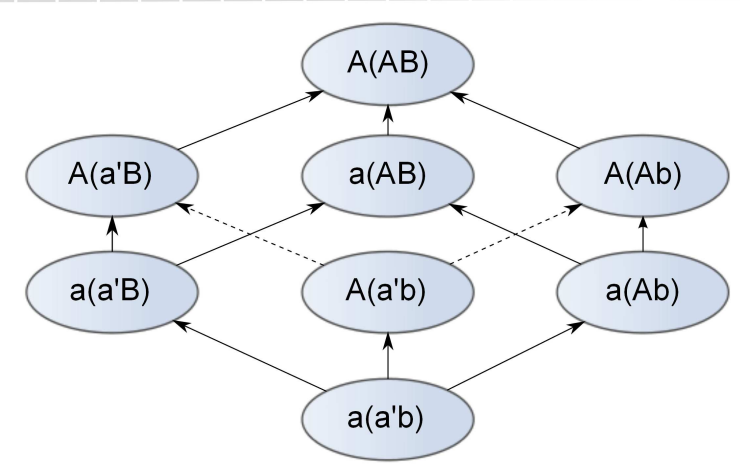

b)

Fig. 2 Hierarchy in sequences

Definition 2.8. The generalized support gen_supp is based on the definition of support in definition 2.2. It's only necessary to redefine the subset relation for two elements to deal with taxonomies upon items. For this purpose we define a generalized subset relation $\subseteq_{g}$ as

$$
\begin{aligned}
e_{1} \subseteq_{g} e_{2} \Leftrightarrow \quad & \forall i \in e_{1}: i \in e_{2} \vee \\
& \exists j \in e_{2}: i \in \operatorname{ancestors}(j) .
\end{aligned}
$$

A sequence $\alpha=\left\langle a_{1} a_{2} \ldots a_{n}\right\rangle$ is a generalized subsequence of a sequence $\beta=\left\langle b_{1} b_{2} \ldots b_{m}\right\rangle$ if there exist integers $1 \leq j_{1}<j_{2}<\cdots<j_{n} \leq m$ such that $a_{1} \subseteq_{g} b_{j_{1}}, a_{2} \subseteq_{g}$ $b_{j_{2}}, \ldots, a_{n} \subseteq_{g} b_{j_{n}}$. We denote $\alpha \sqsubseteq_{g} \beta$. For completeness, the definition of the generalized support of a sequence $s_{1}$ is

$$
\text { gen_supp }\left(s_{1}\right)=\left|\left\{\langle S I D, s\rangle \mid(\langle S I D, s\rangle \in D) \wedge\left(s_{1} \sqsubseteq_{g} s\right)\right\}\right| .
$$

Example 2.4. An example of how the generalized support of a sequence is computed can be shown on a sequence database from table 1 and taxonomy structures over items $a, a^{\prime}, b, b^{\prime}$ from figure 2. For sequence $s_{1}=\langle a(A b)\rangle$ generalized support gen_supp $\left(s_{1}\right)=2$, because it is generalized sequence of tuples with $S I D \in\{1,5\}$. If we take any of the sequence parents of $s_{1}$, the sequence $s_{2}=\langle a(A B)\rangle$, the generalized support will be even higher. In addition to $s_{1}, s_{2}$ is contained in the tuple with $S I D=4$, because $\langle a(A B)\rangle \sqsubseteq_{g}\left\langle b^{\prime} a a\left(a^{\prime} b^{\prime}\right) b\right\rangle$. The higher support is the consequence of $(A B) \subseteq_{g}\left(a^{\prime} b^{\prime}\right)$. Element $(A b) \nsubseteq_{g}\left(a^{\prime} b^{\prime}\right)$, so that is why sequence $s_{1}$ has lower support than $s_{2}$.
Having the definition of the taxonomy structure, we can define the task of mining sequential patterns with taxonomy in almost the same way as the problem without taxonomies. In addition, the presence of taxonomies over the items in a sequence database causes that more general terms will occur in a result.

Table 1 Example of sequence database $D$ with 5 sequences,

$$
|D|=5
$$

\begin{tabular}{|c|c|}
\hline SID & sequence \\
\hline \hline 1 & $\left\langle a b\left(a^{\prime} b\right) b\right\rangle$ \\
\hline 2 & $\left\langle b\left(a a^{\prime}\right) b\left(a b^{\prime}\right) a a\right\rangle$ \\
\hline 3 & $\left\langle(a b) b a^{\prime} a\left(b b^{\prime}\right)\right\rangle$ \\
\hline 4 & $\left\langle b^{\prime} a a\left(a^{\prime} b^{\prime}\right) b\right\rangle$ \\
\hline 5 & $\langle(a b)(a b)(a b)\rangle$ \\
\hline
\end{tabular}

However not all of these patterns are interesting for an analyst. The question is: which of the generalized sequences are interesting to incorporate them into result set of patterns? This decision can be based on information theory which is described in more details in section 4 .

\section{RELATED WORK}

The first algorithm for mining sequential patterns AprioriAll was published by Agrawal and Strikant in 1995 in [2]. It was a modification of the well-known Apriori algorithm for mining association rules such that it can mine sequential patterns. The general idea of both Apriori and AprioriAll is based on a candidate generate-and-test framework. The same authors then presented algorithm Generalized Sequence Patterns (GSP) in [3]. Our proposed algorithm is based on GSP, so this algorithm will be described in more detail.

\subsection{GSP algorithm}

As mentioned, the general idea of this algorithm is based on the Apriori property and candidate generation and testing approach. The Apriori property states: Every nonempty subsequence of a sequential pattern is a sequential pattern [1].

The algorithm works in several phases. In each of them it makes a pass over the sequence database:

1. In the first phase, the support of items is counted and those having it higher than the min_sup are inserted in the resulting set $L_{1}$ containing frequent 1-sequences.

2. Each of the next phases consists of two sub-steps.

(a) At first, in the join step, a candidate set $C_{k}$ is generated from the set of frequent items from the previous phase $L_{k-1}$. The candidate sequences $C_{k}$ contain one more item than sequences in $L_{k-1}$. Candidates of length two are generated from items in the manner that either they occur in one transaction, thus forming an element, or one is after the other, thus forming a sequence. For example, for items $a, b$ either $\langle(a b)\rangle$ or $\langle a b\rangle$ can be generated. Candidates of 
higher length are generated from $s_{1}$ and $s_{2}$ such that if the first item of $s_{1}$ and the last item of $s_{2}$ are omitted, the resultant subsequences are the same. The sequences $s_{1}$ and $s_{2}$ are called contiguous and those $k$-sequences which has nonfrequent contiguous subsequences, are pruned.

(b) After that, in counting step, the support of each candidate is counted resulting in frequent set $L_{k}$. The algorithm terminates in phase $n$ when no candidate sequence satisfies the minimum support condition or no candidate sequence can be generated because of pruning step. The result set is composed of $\bigcup_{k=1}^{n-1} L_{k}$.

In comparison to AprioriAll, GSP incorporates time constraints, sliding time windows and taxonomies in sequence patterns and thus allowing to mine for generalized patterns. The patterns with given time constraints and sliding time windows are achieved by a procedure which detects if the candidate is a subsequence of any sequence in database satisfying the constraints.

\subsection{Other sequence pattern mining algorithms}

Some modifications of the algorithm GSP were published later, for example, PSP in [4] introduces a different tree structure for maintaining candidates. In the category of algorithms based on candidate generating and testing, it's worth mentioning the algorithm SPADE [5] which uses for mining vertical representation of sequence database; and also SPAM [6], which is similar to SPADE but uses internal bitmap structure for database representation.

The next family of algorithms is based on the patterngrowth principle. The key idea of these algorithms is that at first, it is created a representation of the sequence database to partition the space and then the search space is often traversed in depth-first manner to generate less candidate sequences. The most famous representative is the PrefixSpan published by Pei et al. in [7], which uses projected database with respect to some prefix for database representation.

The most recent algorithms are based on early pruning. The algorithms try to prune the searched space in the earliest phases based on the position induction. They store the last positions of items in database sequences and use this information to prune candidates which can't be appended to the current prefix. The rest of the algorithm is the same as in pattern-growth based. LAPIN [8] is the typical representative of these algorithms.

\subsection{Hierarchies in sequence pattern mining}

The authors of GSP presented the way how to incorporate the taxonomies into the process of sequence pattern mining [3]. The idea is based on replacing all the sequences in database with "extended-sequences". In this extended form, in addition to the item, the information of all its ancestors is stored. For example, sequence $\langle($ milk, bread $)$ (croissant $)\rangle$ from the taxonomy in Figure 1 is replaced by $\langle($ milk, pastry, dairy product, food $)$ (croissant, pastry, food $)\rangle$. Then the GSP algorithm is the same as was mentioned before on these "extendedsequences". Although the authors proposed two optimizations, this approach requires much more space for storing the database sequences. It also allows to mine all the frequent sequences, even those which could be uninteresting.

In [9] the authors were using their framework for multidimensional sequence mining. They presented the HYPE algorithm to incorporate hierarchies into this framework and mine for multidimensional sequences over several levels of hierarchy.

In [10] T. Huang presented the concept of fuzzy multilevel sequential patterns. In this concept the item is allowed to belong among more general concepts, for example, a tomato can be considered either as a fruit or a vegetable. This relationship can be represented by a value between 0 and 1 . They proposed the algorithm based on the divide-and-conquer strategy and an efficient algorithm to mine fuzzy cross-level patterns.

\section{HGSP ALGORITHM}

In this section we describe our algorithm $h G S P$ (hierarchical-GSP) for mining multi-level (level crossing) sequence patterns. The algorithm is based on GSP [3]. In contrast to the method based on "extended-sequences", our method tries to reduce the number of redundant patterns. Notice that if a sequence $s$ is frequent and $s_{1} \in$ ancestors $_{\text {seq }}(s)$, then $s_{1}$ is always frequent. It can be observed that $s_{1}$ is redundant because it does not contain any new information. Due to this observation our algorithm does not generate all possible generalizations of all frequent sequences but performs generalization when a sequence would be pruned. hGSP is based on the idea of concreteness of each sequence. The concreteness measure will be evaluated using information theory explained in the following subsection. In following text we use support term in the meaning of our defined generalized support.

\subsection{Concept of hGSP Algorithm}

The main idea of our algorithm is that if a sequence $s$ has support gen_supp $(s)$, there can exist a generalized sequence $s_{g} \in$ parent $_{\text {seq }}(s)$ such that gen_supp $\left(s_{g}\right)>$ gen_supp $(s)$. This can be applied repeatedly. Notice that $\forall s_{g} \in$ parents $_{\text {seq }}(s):$ gen_supp $(s) \leq$ gen_supp $\left(s_{g}\right)$. Unfortunately, during generalization some information is being lost. Because of this, the quality of mined generalized frequent items strictly depends on selection of a generalized sequence from the set of generalized sequences. The concept of information theory is used for this purpose. Generally, we expect that more specific sequence $s$ is more important result than it's generalized form $s_{g}$ because the generalized $s_{g}$ is more expectable in the result set. This corresponds with meaning of information content.

Definition 4.1. The Shannon information content [11] of a value $x$ with probability $p(x)$ is defined as

$h(x)=\log _{2} \frac{1}{p(x)}$. 
The probability $p(s)$ that sequence $s$ occurs in source database $D$ is

$p(s)=\frac{\text { gen_supp }(s)}{|D|}$.

The information content of sequence $s$ in database $D$ is

$h(s)=\log _{2} \frac{1}{\frac{\text { gen_supp }(s)}{|D|}}=-\log _{2} \frac{\text { gen_supp }(s)}{|D|}$.

For a sequence $s$, the dependence between information content $h(s)$ and generalized support gen_supp $(s)$ causes that if the generalization from $s$ to $s_{g}$ is performed and gen_supp $\left(s_{g}\right)>$ gen_supp $(s)$, then $h\left(s_{g}\right)<h(s)$. Therefore the generalization should be performed only if the candidate sequence is not frequent (i.e. gen_supp $(s)<$ min_supp) or the GSP algorithm cannot perform join of two candidate sequences with joinable sequence ancestors.

Definition 4.2. (Concreteness) The sequence $s_{1}$ is more concrete than another sequence $s_{2}$ if $\left(h\left(s_{2}\right)<h\left(s_{1}\right)\right) \wedge$ (ancestors $\left._{\text {seq }}\left(s_{1}\right) \cup s_{1}\right) \cap\left(\right.$ ancestors $\left._{\text {seq }}\left(s_{2}\right) \cup s_{2}\right) \neq \emptyset$.

\subsection{Algorithm details}

The hGSP algorithm uses modified join step and pruning step of the GSP algorithm. The rest of the algorithm remains the same. With no loss of generality, in our implementation we assume that items in an element of a sequence are in lexicographic order (similarly as authors in [3]).

The join step is modified for generating candidates of length $k=3$ and more. Let's have a pair of frequent sequences $s_{1}$ and $s_{2}$ of length $k-1$. A join can be performed if subsequences of $s_{1}$ after omitting the first item and $s_{2}$ after omitting the last one have a common sequence ancestor. Then the joined sequence of length $k$ is composed from the first item of $s_{1}$, the most concrete sequence ancestor of common part and the last item of $s_{2}$. The last item is added the same as in GSP.

The support of candidates is counted in the same way as in the original GSP. The only difference is that we use gen_supp $(s)$ defined in Definition 2.8 instead of common support. Therefore, only the procedure for checking, if a candidate is subsequence of a given database, is modified.

A modification of the pruning step is shown in Algorithm 1. The algorithm uses a method for finding the approximation of the most concrete generalization set of a sequences which is described in Algorithm 2. Our approximation algorithm is based on the greedy optimization technique. As a result, the algorithm finds only sequences corresponding to the local optimum of concreteness measure. Finding of a global optimum would be extremely computationally complex because of a large search-space.

During generalization, generalized sequences that contain ancestor item of another item in the same element can occur. Such sequences are redundant and they are discarded.

We have formulated a theorem that it is not necessary to evaluate information content using logarithm functions but it is sufficient to compare ratios of supports of sequences and theirs generalized forms.

Let's have a sequence $s$ and its generalized form $s_{1}$. The information contents of these sequences are $h(s)=$ $-\log _{2} \frac{\text { gen_supp }(s)}{|D|}$ and $h\left(s_{1}\right)=-\log _{2} \frac{\text { gen_supp }\left(s_{1}\right)}{|D|}$. The information lost during generalization of $s$ to $s_{1}$ is $\Delta h=$ $h(s)-h\left(s_{1}\right)$. It follows that

$\Delta h=\log _{2}\left(\frac{\frac{g \text { gen_supp }\left(s_{1}\right)}{|D|}}{\frac{g e n \_ \text {supp }(s)}{|D|}}\right)=\log _{2}\left(\frac{\operatorname{gen} \_\operatorname{supp}\left(s_{1}\right)}{g e n \_s u p p(s)}\right)$.

The generalization of $s$ with the smallest information loss is found because then the sequences will be the most concrete. Therefore the algorithm minimizes ratio $\frac{\text { gen_supp }\left(s_{1}\right)}{\text { gen_supp }(s)}$.

\section{Algorithm 1: Method find_generalization()}

Input: A candidate sequence $s$

Output: A set of the most concrete generalizations $G_{s}$.

Method:

$$
\begin{aligned}
& G_{s}=\{\} ; \\
& \text { min_supp_ratio }=+\infty ; \\
& \text { foreach }\left(p_{s} \in \text { parent } s_{\text {seq }}(s)\right) \text { do: } \\
& \quad \text { ratio }=\text { gen_supp }\left(p_{s}\right) / g e n \_s u p p(s) ; \\
& \text { if }\left(\text { gen_supp }\left(p_{s}\right)<>\text { gen_supp }(s)\right. \\
& \quad \wedge \text { ratio }<\text { min_supp_ratio }) \text { then } \\
& \quad G_{s}=\left\{p_{s}\right\} ; \\
& \quad \text { min_supp_ratio }=\text { ratio; } \\
& \text { elseif }(\text { ratio }=\text { min_supp_ratio }) \text { then } \\
& \quad G_{s}=G_{s} \cup\left\{p_{s}\right\} ; \\
& \text { endif; } \\
& \text { endforeach; } \\
& \text { return } G_{s} ;
\end{aligned}
$$

\section{Algorithm 2: hGSP Pruning Step}

Input: A set of candidates $-C_{k}$, minimal support min_supp Output: A set of frequent sequences $L_{k}$ of length $k$

\section{Method:}

$$
\begin{aligned}
& L_{k}=\{\} \\
& \text { foreach } s_{c} \in C_{k} \text { do: } \\
& C_{k}^{\prime}=\left\{s_{c}\right\} \\
& \text { sequence_added }=\text { false; } \\
& \text { while }\left(\text { sequence_added }=\text { false } \wedge\left|C_{k}^{\prime}\right|>0\right) \text { do: } \\
& \quad G_{s}=\{\} ; \\
& \quad \text { foreach } s \in C_{k}^{\prime} \text { do: } \\
& \quad \text { if } \text { gen_supp }(s) \geq \text { min_supp then } \\
& \quad L_{k}=L_{k} \cup\{s\} \\
& \quad \text { sequence_added }=\text { true; } \\
& \quad \text { else } \\
& \quad G_{s}=G_{s} \cup \text { find_generalization }(s) \\
& \quad \text { endif; } \\
& \quad \text { endforeach; } \\
& \quad C_{k}^{\prime}=G_{s} ; \\
& \text { endwhile; } \\
& \text { endforeach; } \\
& \text { return } L_{k} ;
\end{aligned}
$$




\section{EXPERIMENTS}

We have used synthetic datasets created by the generator described in [12] with our hierarchical extension. The evaluation was performed on PC Intel Core Duo 2.66 GHz, 2GB RAM, OS Windows XP 32-bit. The implementation of the hGSP algorithm was integrated into Microsoft SQL Server 2008 R2 Analysis Services.

\subsection{Performance evaluation}

A performance test is focused on the execution time of the algorithm. We did not compare execution times with GSP because hGSP generates much more candidates. Also, GSP generates shorter sequences and it is finished after sequences of length 2 in most cases. Therefore, we show only execution times of hGSP. Parameters of datasets were:

- average DB sequence length $=25$,

- hierarchy count $=25$,

- average hierarchy depth $=3$,

- frequent sequences count $=0.2 \%$ of $|D|$

- and average length of frequent sequences $=7$.

Results of the experiment are shown in Figure 3. The algorithm was executed on datasets of a different size and the execution time was measured. The number of transaction items ranged from 25,000 in a database of 1,000 sequences up to 225,000 transaction items in a database of 9,000 sequences. The plot shows that processing time increases linearly with the dataset size because the algorithm checks if each candidate sequence is subsequent of any database sequence.

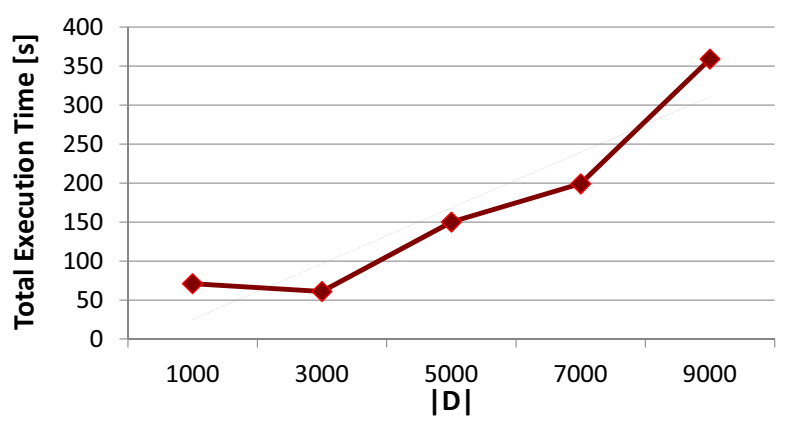

Fig. 3 Average processing time of one candidate sequence

\subsection{Generalization evaluation}

A generalization evaluation experiment is focused on the generalization property of the hGSP algorithm. The methodology of the evaluation is following. It is known that the number of frequent sequences in results strictly depends on the minimal support value. Therefore, we have compared the number of candidates and mined frequent sequences for different values of the minimal support. The results were measured for relative supports from $20 \%$ up to $80 \%$. Predefined parameters of frequent sequences in the dataset were: the average length 5 and support $50 \%$. Results of hGSP are shown in a comparison to GSP results.

The results in Figure 4 show that the number of candidate/frequent sequences increases exponentially with descending value of minimal support for both hGSP and GSP. In general, hGSP creates more than 10 times more frequent sequences than GSP because of the generalization property. This is the main substance for analyst - hGSP does not prune important candidate sequences if there is a possibility to generalize them. In addition, Figure 5 shows that hGSP creates sequences with more items than GSP.

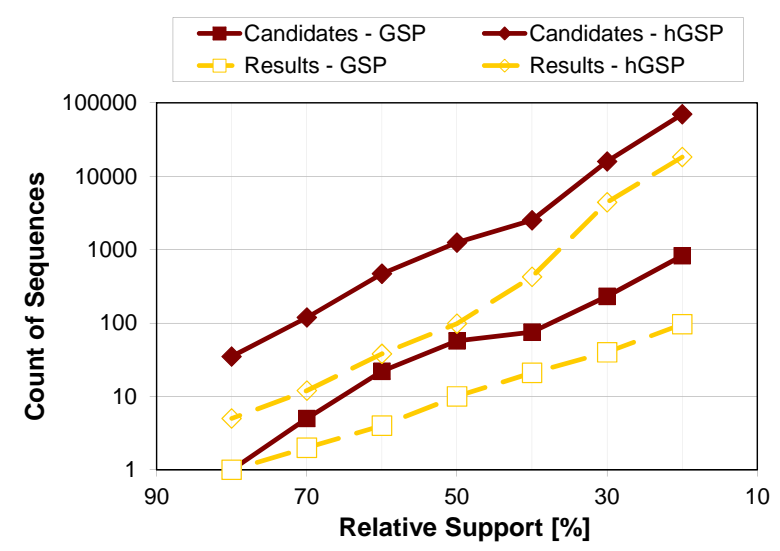

Fig. 4 Numbers of candidate and frequent sequences with dependency on minimal support

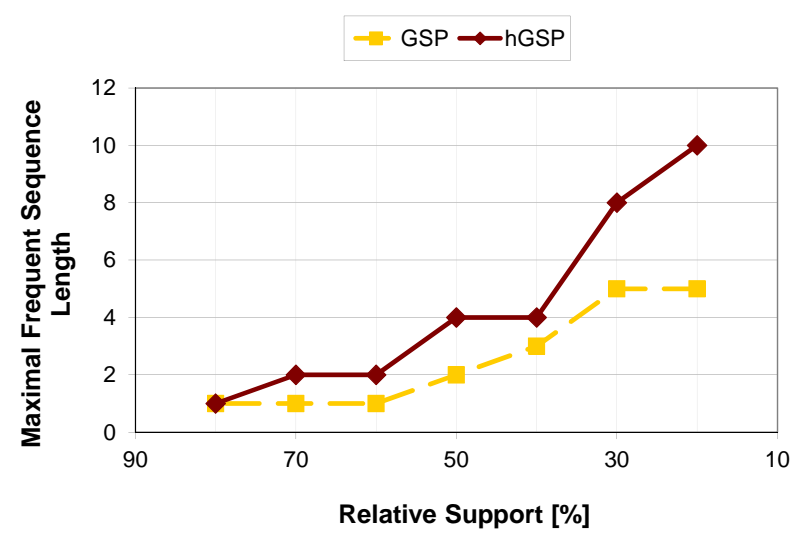

Fig. 5 Lengths of frequent sequences with dependency on minimal support

\subsection{Comparison of the hGSP algorithm and GSP using "extended-sequences"}

Experiments show a dependency of execution time and the number of patterns on a different number of frequent se- 
quential patterns in a database of length $7\left|L_{7}\right|$. The hGSP algorithm is compared with a recommended taxonomy extension of GSP based on "extended-sequences" (see section 3.3) without using optimizations. Parameters of the datasets were transactions count $=5000$, hierarchy count $=15$, average hierarchy depth $=5$, other parameters were the same as in the experiment in Section 5.1.

In Table 2 it is shown that hGSP is faster up to $10 \%$ than GSP using "extended-sequences". The main disadvantage of the GSP approach is shown in Table 3. GSP using "extended-sequences" generates a huge amount of redundant (candidate and frequent) sequences and the result has to be filtered, suggested author's optimization reduces the amount but the result set still contains many redundant patterns.

Table 2 Execution times of hGSP and GSP in seconds

\begin{tabular}{|c|c|r|}
\hline$\left|L_{7}\right|$ & hGSP & GSP \\
\hline \hline 25 & $1536 \mathrm{~s}$ & $1546 \mathrm{~s}$ \\
50 & $3968 \mathrm{~s}$ & $4229 \mathrm{~s}$ \\
100 & $1327 \mathrm{~s}$ & $1515 \mathrm{~s}$ \\
\hline
\end{tabular}

Table 3 Total sum of sequential patterns of all lengths $\left|\bigcup_{k \geq 1} L_{k}\right|$

\begin{tabular}{|c|r|r|}
\hline$\left|L_{7}\right|$ & hGSP & GSP \\
\hline \hline 25 & 3329 & 13350 \\
50 & 7750 & 33611 \\
100 & 4187 & 11316 \\
\hline
\end{tabular}

\section{CONCLUSIONS}

In this paper we presented a new way how to incorporate taxonomies into the process of mining generalized sequential patterns. Our approach is based on information theory and modifies the well known the GSP algorithm. The algorithm is trying to generalize sequences only if it is required. This is because of either low support of the sequence or inability to join two sequences with joinable sequence ancestors. In the experiments, we showed that in comparison to GSP, the algorithm was able to find more patterns and patterns of higher length thanks to the generalization.

In the future work, we want to focus on optimization of the algorithm. Because hGSP generates more candidates, it is naturally slower than GSP but outperforms the approach of extended databases for mining patterns with taxonomies. Because of inefficient candidates generating and testing we will also try to apply our ideas to more efficient algorithm such as PrefixSpan.

\section{ACKNOWLEDGEMENT}

This work has been supported by the research funding TAČR TA01010858, BUT FIT grant FIT-S-11-2, the Research Plan No. MSM 0021630528 and the IT4Innovations Centre of Excellence CZ.1.05/1.1.00/02.0070. The authors would like to thank Zbyněk Křivka for his valuable suggestions and advices concerning mathematical definitions. The authors would also like to thank the anonymous reviewers for their valuable comments.

\section{REFERENCES}

[1] HAN, J. - KAMBER, M.: Data mining: concepts and techniques. 2nd ed., Elsevier, 2006.

[2] AGRAWAL, R. - SRIKANT, R.: Mining sequential patterns. In Proceedings of the Eleventh International Conference on Data Engineering, ICDE '95, pp. 3-14, 1995, Washington, DC, USA. IEEE Computer Society.

[3] SRIKANT, R. - AGRAWAL, R.: Mining sequential patterns: Generalizations and performance improvements. In Proceedings of the 5th International Conference on Extending Database Technology: Advances in Database Technology, EDBT '96, pp. 3-17, London, UK, 1996. Springer-Verlag.

[4] MASSEGLiA, F. - CATHALA, F. - PONCELET, P.: The PSP approach for mining sequential patterns. In Proceedings of the Second European Symposium on Principles of Data Mining and Knowledge Discovery, PKDD '98, pp. 176-184, London, UK, 1998. SpringerVerlag.

[5] ZAKI, M. J.: Spade: An efficient algorithm for mining frequent sequences. In Machine Learning, Vol. 42, No. 1/2, pp. 31-60, January 2001.

[6] AYRES, J. - FLANNICK, J. - GEHRKE, J. - YIU, T.: Sequential pattern mining using a bitmap representation. In Proceedings of the eighth ACM SIGKDD international conference on Knowledge discovery and data mining, KDD '02, pp. 429-435, New York, NY, USA, 2002. ACM.

[7] PEI, J. - HAN, J. - MORTAZAVI-ASL, B. - WANG, J. - PINTO, H. - CHEN, Q. - DAYAL, U. - HSU, M.: Mining sequential patterns by pattern-growth: The PrefixSpan approach. IEEE Trans. on Knowl. and Data Eng., pp. 1424-1440, November 2004.

[8] YANG, Z. - WANG, Y. - KITSUREGAWA, M.: Lapin: effective sequential pattern mining algorithms by last position induction for dense databases. In Proceedings of the 12th international conference on Database systems for advanced applications, DASFAA'07, pp. 1020-1023, Berlin, Heidelberg, 2007. Springer-Verlag.

[9] PLANTEVIT, M. - LAURENT, A. - TEISSEIRE, M.: HYPE: mining hierarchical sequential patterns. In DOLAP 2006, ACM 9th International Workshop on Data Warehousing and OLAP, pp. 19-26, November 2006.

[10] HUANG, T. Ch.: Developing an efficient knowledge discovering model for mining fuzzy multi-level sequential patterns in sequence databases. Fuzzy Sets Syst., 160:3359-3381, December 2009.

11] MACKAY, D.: Information Theory, Inference, and Learning Algorithms. Cambrifge University Press, 2003. 
[12] AGRAWAL, R. - SRIKANT, R.: Fast algorithms for mining association rules. In Proc. of the VLDB Conference., pp. 487-499, Santiago, Chile, 1994. Expanded version available as ABM Research Report RJ8939.

[13] SHIN-ICHI, N.: Efficient generation of plane trees. In Inf. Process. Lett., Vol. 86, No. 3, pp. 167-172, November 2002 .

Received January 31, 2012, accepted June 14, 2012

\section{BIOGRAPHIES}

Michal Šebek is $\mathrm{PhD}$ student at Faculty of Information Technology, Brno University of Technology. He received his master's degree in Computer Science in 2009. His master theses was focused on development of open-source data mining system FIT-Miner. His PhD research focuses on knowledge discovery from data streams.

Martin Hlosta is a second year PhD student. He received his master's degree in Computer Science in 2010 from Faculty of Information Technology, Brno University of Technology. Both his bachelor and master theses were focused on methods of knowledge discovery in databases. His $\mathrm{PhD}$ research focuses on knowledge discovery from malware detection data.
Jan Kupčík received his master's degree in Information Systems from Faculty of Information Technology, Brno University of Technology in 2007. He is currently pursuing the $\mathrm{PhD}$ degree at the same university. His research interests include database and OLAP technologies, data mining and software engineering.

Jaroslav Zendulka received his M.Sc. degree in computers and Ph.D. in technical cybernetics at the Brno University of Technology, Czech Republic. He is currently an Associate Professor at the Department of Information Systems at the Brno University of Technology. He has participated in several projects and has written tens of papers in international journals and conference proceedings. $\mathrm{He}$ is a PC member of several international conferences. His research interests include data and object modelling; database technology and information systems; data mining.

Tomáš Hruška received his Ing. (MSc.) and CSc. (PhD) titles from Brno University of Technology, Czech Republic. Since 1978 works at the Brno University of Technology, since 1998 as full professor. In 1978-1983, he dealt with research in the area of compiler implementation for simulation languages. He dealt with an implementation of an object-oriented database systems as a tool for modern information systems design and Lissom/Codasip project now. It is focused on the research of the processor description language for transformations of processor models. 